\title{
AVALIAÇÃO DA RESILIÊNCIA: UMA REVISÃO INTERNACIONAL
}

RESILIENCE ASSESSMENT: AN INTERNATIONAL REVIEW

EVALUACIÓN DE LA RESILIENCIA: UNA REVISIÓN INTERNACIONAL

\author{
Karina da Silva Oliveira* \\ Tatiana de Cássia Nakano**
}

\begin{abstract}
RESUMO
O termo resiliência tem sido utilizado para referir-se às condutas assumidas pelos indivíduos ante condições adversas. Há cerca de 40 anos, pesquisadores têm buscado compreender o fenômeno e propor formas de avaliação dessa característica. O objetivo deste estudo foi compreender de que forma pesquisadores internacionais têm abordado a questão da avaliação da resiliência. Para isso, a revisão da literatura científica foi feita junto a três bases eletrônicas de dados (Academic Search Premier, Academic Search Elite e Medline Complete), nas quais foram selecionadas 68 publicações. Os dados indicaram predominância de estudos empíricos, voltados a adultos, sendo a construção de instrumentos tomada como temática mais frequente $(35,29 \%), 55$ diferentes técnicas foram usadas como formas de aferir o construto, sendo somente 10 relacionadas diretamente à resiliência. É possível concluir que a avaliação da resiliência é um tema em desenvolvimento internacionalmente e que apresenta relevância para o cenário científico.
\end{abstract}

Palavras-chave: Avaliação. Resiliência psicológica. Revisão de literatura. Testes psicológicos.

\begin{abstract}
Resilience has been frequently associated with the ability to bounce back onto the face of adversity. For about 40 years, researchers have been longing, not only, to understand this phenomenon, but also, to develop proper measurement instruments. The aim of this study is to understand how international researchers have addressed the issue of resilience assessment. Sixty-eight (68) articles were identified out of three electronic databases (Academic Search Premier, Academic Search Elite and Medline Complete) which were used to carry out the literature review. The data
\end{abstract}

Texto recebido em 9 de outubro de 2016 e aprovado para publicação em 28 de março de 2017.

* Doutoranda em Psicologia como Profissão e Ciência pela Pontifícia Universidade Católica de Campinas (PUC Campinas), bolsista CAPES. Endereço: Avenida John Boyd Dunlop, s/nº - Jardim Ipaussurama, Campinas-SP, Brasil. CEP: 13060-904.

Telefone: (19) 3343-6892, fax: (19) 3343-6891. E-mail: karina_oliv@yahoo.com.br.

** Docente no Curso de Pós-Graduação em Psicologia como Profissão e Ciência da PUC Campinas. 
indicated predominance of empirical studies focused on adults, and the most frequent topic was the development of instruments (35.29\%), 55 different instruments were cited as ways of assessing resilience, but only 10 of them were directly related to resilience. It is possible to say that resilience assessment is an internationally growing theme, and it is also a relevant topic within the scientific scenario.

Keywords: Assessment. Psychological resilience. Review literature. Psychological test.

\section{RESUMEN}

El término resiliencia ha sido utilizado para referirse a comportamientos asumidos por personas que enfrentan condiciones adversas. Durante aproximadamente 40 años, los investigadores han tratado de comprender el fenómeno y proponer formas de evaluar esta función. El objetivo de este trabajo es conocer cómo los investigadores internacionales han abordado el tema de la evaluación de la resiliencia. La revisión de la literatura científica fue realizada con tres bases de datos electrónicas (Academic Search Premier, Academic Search Elite y Medline completa), siendo seleccionadas 68 publicaciones entre los años 2001 y 2016. Se identificó un predominio de estudios empíricos centrados en adultos; la construcción de instrumentos fue considerada el tema más frecuente $(35,29 \%)$. Un total de 55 técnicas diferentes fueron usadas, siendo solamente 10 relacionadas directamente con la capacidad investigada. Se concluyó que la evaluación de la resiliencia es un tema en desarrollo a nivel internacional y que tiene relevancia en la escena científica.

Palabras clave: Evaluación. Resiliencia psicológica. Revisión de literatura. Construcción de herramientas.

\section{INTRODUÇÃO}

reação dos indivíduos diante das adversidades tem sido tema de investigação
na Psicologia desde a década de 1970 (Prince-Embury, Saklofske, \& Keefer,
2017), tendo como foco o conceito de invulnerabilidade, notadamente voltado à identificação dos fatores de risco e das adversidades que um indivíduo era capaz de suportar (Brandão, Mahfoud, \& Gianordoli-Nascimento, 2011). Essa tendência perdurou durante a década de 1980, quando programas de prevenção e intervenção com as populações de risco começaram a ser elaborados (Sapienza \& Pedromônico, 2005), o termo invulnerabilidade era o mais utilizado por esses primeiros autores. Posteriormente, por volta da década de 1990, uma mudança no termo invulnerabilidade acabou por dar origem às primeiras 
avaliações relacionadas ao termo resiliência, as quais tinham como alvo conhecer e discriminar o quanto um indivíduo era capaz de suportar condições adversas (Pesce, Assis, Santos, \& Oliveira, 2004). Ressalta-se, nesse sentido, que as formas de avaliação da resiliência não contemplavam aspectos positivos, assim como a amplitude de possibilidades de expressão e manifestação do potencial resiliente.

Percebe-se que o percurso histórico da investigação da resiliência acompanhou uma tendência que perdurou durante muito tempo na Psicologia, a qual, tradicionalmente, buscou a compreensão das psicopatologias, construindo, ao longo de sua história, um grande leque de conhecimentos teóricos e empíricos, voltados ao desenvolvimento das condições patológicas e da eficácia das terapias para o enfrentamento das enfermidades (Greco, Morelato, \& Ison, 2006). Tal ênfase sobre os aspectos psicopatológicos ocasionou em uma lacuna teórica em relação aos aspectos positivos e aos recursos internos dos indivíduos, tais como o bem-estar, a satisfação, a esperança, o otimismo, a resiliência, entre outros (Seligman \& Csikszentmihalyi, 2000), de modo que, como consequência, fez-se notar, até mais recentemente, uma compreensão limitada acerca dos benefícios que tais características poderiam conferir aos indivíduos.

Como reação, o movimento denominado Psicologia Positiva surgiu e tem, ao longo das últimas décadas, se consolidado, amparado em uma proposta de alteração de postura e de paradigma ante as possibilidades de atuação profissional e de pesquisa (Yunes, 2003). Dentro dele, é possível observar o esforço de inúmeros profissionais em contribuir para a construção do conhecimento voltado aos fenômenos positivos, indicativos de "vida saudável", relacionados diretamente aos sistemas de adaptação ao longo do desenvolvimento, entre os quais se pode destacar a resiliência (Masten, 2014).

Como consequência, o conceito de resiliência passou a receber atenção por parte dos pesquisadores, sendo compreendido como um processo que confere ao indivíduo habilidades para lidar com as adversidades experimentadas, por meio da intersecção de aspectos sociais, habilidades e potencialidades individuais (Grego, Morelato, \& Ison, 2006; Junqueira \& Deslandes, 2003). Assim, faz-se notar que a resiliência se caracteriza por ser um termo ainda em desenvolvimento e fase de consolidação, mas que tem, cada vez mais, despertado o interesse da comunidade científica (Prince-Embury et al., 2017; Sims \& Pooley, 2017).

Ainda que não se faça notar a presença de uma definição que seja amplamente aceita pelos pesquisadores, algumas tentativas de sintetizar as diferentes propostas de conceituação do termo foram elaboradas e envolvem algumas características, notadamente a compreensão da resiliência como um fenômeno psicológico (Yunes, 2003) que está presente em todos os indivíduos (Masten, 2001; 2014), 
que pode desenvolver-se e manifestar-se ao longo do ciclo vital (Fontes, 2010; Godoy, Joly, Piovezan, Dias, \& Silva, 2010; Infante, 2007; Masten, 2001; Poletto, Wagner, \& Koller, 2004), sempre que o indivíduo for submetido a uma condição adversa (Luthar, Cicchetti, \& Becker, 2000; Yunes, 2011).

Como condição adversa, a literatura tem feito a distinção entre condição adversa real ou percebida (Castillo, Castillo-López, López-Sánches, \& Dias, 2016), cujo enfrentamento envolverá a utilização de recursos sociais (Masten, 2001, 2014; Prince-Embury, 2006; 2010; Yunes, 2011) e individuais (Castillo et al., 2016; Gloria \& Steinheardt, 2014), esperando-se que resulte em um desfecho positivo, ou ainda, adaptação positiva (Infante, 2007; Masten, 2001; Reppold, Mayer, Almeida, \& Hutz, 2012).

Dada a sua complexidade, algumas recomendações acerca dos cuidados que devem permear a avaliação da resiliência têm sido enfatizadas e envolvem a necessidade que, durante o processo de mensuração dessa característica, aspectos relacionados a adversidades, tais como os riscos e eventos negativos experimentados em diferentes níveis (biológicos, físicos, sociais, emocionais e desenvolvimentais) sejam considerados (Masten, 2001). Torna-se fundamental, nesse processo avaliativo, compreender de que maneira características internas, cognitivas e emocionais podem influenciar na resposta resiliente de um indivíduo (Infante, 2007), assim como o conhecimento de aspectos relacionados à adaptação positiva disponíveis, atuantes como fatores de proteção, considerando-os em todos os níveis citados.

Dada a relevância que a resiliência e seu estudo têm demonstrado na Psicologia (Masten, 2014; Prince-Embury et al., 2017; Yunes, 2003), um investimento em ferramentas para sua avaliação se mostra essencial, notadamente aqueles voltados à construção e validação de instrumentos que considerem o termo como multidimensional, visto como um processo dinâmico e não somente como um conjunto de indicadores de competência e ajustamento biopsicossocial (Reppold et al., 2012). Isso porque, ainda segundo os autores, a mensurabilidade psicológica da resiliência é um tema bastante controverso e tradicionalmente é feito por meio dos escores de testes, medidas de desempenho, como notas escolares, perfis obtidos por questionários de personalidade ou temperamento, por entrevistas e análise de história de vida, ou ainda, de análise de discurso de terceiros que convivem com o indivíduo resiliente. Desse modo,

Infelizmente, a generalidade dos instrumentos que se destinam à avaliação do construto resiliência centra-se em variáveis psicológicas envolvidas na resiliência (como apoio social, coping, autoeficácia, ajustamento emocional, etc.), mas não propriamente no construto em si (Reppold et al., 2012, p. 252). 
Outras críticas relacionadas à sua avaliação envolvem a constatação de que escalas que se propóem a avaliar a resiliência como um traço de personalidade não são eficazes na tarefa da avaliação (Waaktaar \& Torgersen, 2010). Ambos os pontos devem ser considerados como relevantes para o desenvolvimento do tema, pois a avaliação adequada de um conceito psicológico é um procedimento fundamental para a tomada de decisão, especialmente no processo de construção de instrumentos (Prince-Embury et al., 2017).

Assim, diante do exposto e das lacunas que ainda permanecem em relação à temática, este estudo teve como objetivo compreender de que forma os pesquisadores internacionais têm abordado a questão da avaliação da resiliência, por meio da revisão da literatura científica. A relevância desse tipo de estudo deve-se à constatação de que a análise das produções científicas sobre uma determinada área permite a clarificação dos assuntos que têm recebido maior destaque entre os pesquisadores, do tipo de investigação que vem sendo privilegiado, dos instrumentos mais utilizados e da qualidade e efetividade dessas investigações (Méis \& Letas, 1996; Witter, 1999). Do mesmo modo, possibilitam a identificação de lacunas que necessitam de maiores investigações, pois fornecem um mapeamento das contribuições, necessidades e déficits da produção (Ferreira, 2002; Lustoza, Oliveira, \& Mello, 2010).

\section{MÉTODO}

Três bases de dados internacionais foram consultadas, sendo elas a Academic Search Premier, a Academic Search Elite e a Medical Literature Analysis and Retrieval System Online (Medline Complete), as quais foram acessadas por meio da EBSCOHost. As bases de dados foram acessadas no período compreendido entre $1^{\circ}$ de dezembro de 2015 e 23 de março de 2016, tendo sido analisados os trabalhos publicados até essa data, sendo importante salientar que nenhum período específico para a busca foi delimitado.

Com base nos descritores resilience e assessment, utilizados de forma combinada, 480 artigos foram identificados, todavia 237 publicações foram excluídas, pois tratavam-se de duplicatas. Também foram excluídos os trabalhos que enfocavam, em seu conteúdo, apenas um dos temas. A partir desses critérios foram selecionados 68 artigos sendo que, destes, 41 são provenientes da base Academic Search Premier, 24 da Medline Complete e 3 da Academic Search Elite. Cabe informar que o trabalho mais antigo foi datado de 2001, e o mais recente, de 2016. Assim, foram analisados 15 anos de publicações voltadas ao tema. 
Os trabalhos foram analisados considerando o ano da publicação, país, tipo de estudo, população estudada, sendo também categorizados quanto ao tipo de enfoque dado à avaliação da resiliência em cada estudo: construção de instrumento (envolvendo os estudos voltados à elaboração de testes, questionários e inventários para a avaliação da resiliência), risco e prevenção (trabalhos que tiveram como foco as condições possíveis e risco e os potenciais recursos de enfrentamento utilizados pelos indivíduos), promoção da saúde mental (estudos que envolveram a avaliação do potencial resiliente, tomado como estratégia de enfrentamento das condições psiquiátricas), resiliência comunitária (voltados à avaliação do fenômeno em comunidades que enfrentam condições adversas) e avaliação da resiliência em militares (nessa população específica).

Ainda no que diz respeito às análises realizadas, buscou-se compreender de que maneira os pesquisadores têm medido o potencial resiliente em seus estudos. Para isso foi realizado um levantamento dos instrumentos utilizados nos estudos, tomando-se, como critério, a necessidade de que apresentassem, objetivamente, o termo resiliência em seu título. Após identificados e selecionados, aspectos como título do instrumento, autores e ano de publicação, população-alvo, tipo de instrumento, objeto de avaliação e tipo de estudo psicométrico realizado foram analisados nessas publicaçôes.

\section{RESULTADOS E DISCUSSÃO}

Tendo como objetivo compreender o interesse científico voltado à avaliação da resiliência, a primeira análise realizada referiu-se à distribuição dos trabalhos considerando-se o ano da publicação. Os resultados apontaram que a primeira publicação internacional voltada à avaliação da resiliência ocorreu em 2001. Observa-se que o período compreendido entre essa publicação e a mais recente encontrada (2016) proporcionou uma análise das publicações de 15 anos. Se estimarmos uma média de artigos por ano, observa-se que foram publicados 4,53 trabalhos por ano ao longo desse período.

Figura 1 - Frequência das produções internacionais por ano de publicação

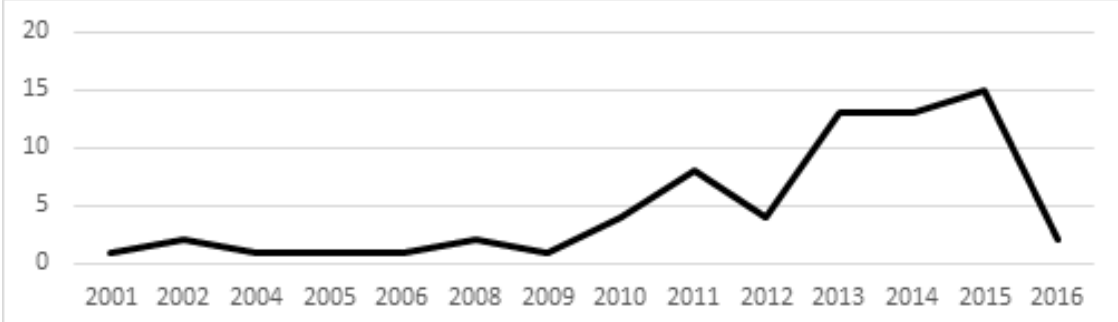

Fonte: elaborado pelas autoras. 
Pela figura 1, nota-se que há uma certa estabilidade no número de publicações entre os anos de 2001 a 2009, em um nível bem baixo. O crescimento no interesse científico, analisado por meio do número de publicações encontradas, tem início a partir de 2010, desde o qual três picos podem ser observados, nos anos de 2011, 2013, sendo o mais alto em 2015. Cabe um apontamento no que se refere às publicações do ano de 2016, pois, conforme se observa na figura 1, há um declínio importante. Todavia é necessário relembrar que o levantamento considerou os artigos publicados até 23 de março de 2016, de modo que demais publicações ocorridas nesse ano não foram analisadas. Por esse motivo, recomenda-se cautela na interpretação desse dado. Os achados confirmam a percepção de Souza e Cerveny (2006), de que a quantidade de artigos publicados sobre resiliência vem crescendo, de maneira considerável, quase triplicando a cada cinco anos.

Possíveis hipóteses explicativas desse movimento envolvem o fortalecimento da compreensão da resiliência como um potencial humano em período imediatamente anterior ao crescimento notado a partir de 2010 (Greco et al., 2006; Junqueira \& Deslandes, 2003; Masten, 2001; Yunes, 2003), bem como o fortalecimento do movimento da Psicologia Positiva, já que é possível notar picos de publicação em períodos próximos ao fortalecimento desse paradigma psicológico (Krentzman, 2013; Pacico \& Bastianello, 2014; Seligman \& Csikszentmihalyi, 2000). Como consequência, o crescimento do interesse dos pesquisadores na mensuração de constructos voltados aos aspectos positivos da experiência humana se fez notar (Pacico \& Bastianello, 2014). Assim, é possível que os picos observados na figura 1 estejam relacionados a esses fatos apresentados na literatura.

O segundo dado analisado referiu-se ao levantamento dos países que têm contribuído com trabalhos voltados à temática da avaliação da resiliência no período analisado. Observou-se que, ao todo, 24 diferentes países tiveram estudos publicados na temática: África do Sul $(\mathrm{n}=2)$, Argentina $(\mathrm{n}=1)$, Austrália $(\mathrm{n}=5)$, Bélgica $(\mathrm{n}=1)$, Canadá $(\mathrm{n}=5)$, Chile $(\mathrm{n}=1)$, China $(\mathrm{n}=2)$, Coreia do Sul $(\mathrm{n}=2)$, Espanha $(\mathrm{n}=3)$, Estados Unidos $(\mathrm{n}=24)$, França $(\mathrm{n}=1)$, Holanda $(\mathrm{n}=2)$, Índia $(\mathrm{n}=1)$, Inglaterra $(\mathrm{n}=6)$, Irã $(\mathrm{n}=1)$, Israel $(\mathrm{n}=1)$, Itália $(\mathrm{n}=1)$, Japão $(\mathrm{n}=1)$, Nigéria $(\mathrm{n}=1)$, Noruega $(\mathrm{n}=1)$, Rússia $(\mathrm{n}=1)$, Suécia $(\mathrm{n}=2)$, Suíça $(\mathrm{n}=1)$ e Taiwan $(\mathrm{n}=1)$.

O país que mais se destacou em publicações relacionadas à avaliação da resiliência foram os Estados Unidos, e também se observa um interesse de outros países nessa temática, ainda que a publicação não seja tão expressiva. Em especial, nos Estados Unidos, Masten (2014) aponta a necessidade objetiva de enfrentamento a adversidades, considerando que o país experimenta, 
continuamente, dificuldades climáticas relacionadas a tornados e furacões, assim como atentados terroristas. Ainda nessa perspectiva, Sims e Pooley (2017) ampliam a discussão para além da realidade americana e trazem à reflexão inúmeras situações de guerra, como a superação necessária para que refugiados retomem suas vidas. Essas considerações, além de justificarem a grande quantidade de países interessados no tema da avaliação da resiliência, também apontam a relevância do tema para a investigação em Psicologia.

Tal quadro pode ser compreendido notadamente se verificarmos que o estudo da resiliência e sua consequente avaliação tem apresentado relevância nas últimas décadas, devido a inúmeras condições adversas e tragédias que têm afligido diferentes comunidades ao redor do globo. Segundo Masten (2014), algumas dessas condições, tais como atentados terroristas, terremotos, tsunamis, entre outras calamidades, ocorridas nos Estados Unidos, Europa, Américas Central e do Sul, assim como na Ásia, provavelmente têm gerado um movimento investigativo sobre suas consequências. Dessa forma, é possível inferir que as produções encontradas nesses países apresentem o movimento científico de pesquisadores em oferecer suporte a indivíduos que enfrentaram, por exemplo, essas adversidades.

Do ponto de vista das populações investigadas pelos estudos, observou-se que $52,94 \%$ dos trabalhos tinham como objeto de investigação a avaliação da resiliência em adultos $(n=36), 17,65 \%$ se propuseram a avaliar a resiliência em crianças $(n=12), 16,18 \%$ em adolescentes $(n=11), 11,76 \%$ referiram-se à avaliação da resiliência em grupos familiares $(n=8)$ e, finalmente, $1,47 \%$ dos trabalhos tinha como intenção de estudo a avaliação da resiliência em idosos $(\mathrm{n}=1)$. Ainda que diversos autores defendam que a resiliência seja um fenômeno que se expressa e se desenvolve ao longo do ciclo vital (Fontes, 2010; Godoy et al., 2010; Infante, 2007; Masten, 2001; Poletto et al., 2004; Yunes, 2011), os estudos analisados neste trabalho apontam para o fato de que, ainda que não distribuídos de forma equilibrada, observou-se o interesse científico sobre o tema nos diferentes momentos do desenvolvimento humano. Entretanto cabe apontar que a avaliação da resiliência em adultos caracteriza-se como de maior interesse por parte dos pesquisadores, ainda que uma ampla variedade de populaçõos venha sendo estudada (população em geral, sobreviventes de traumas diversos, adolescentes, idosos, pacientes em tratamento para estresse pós-traumático, membros de diferentes etnias e culturas), tal como apontado por Lopes e Martins (2011).

Tais dados ainda confirmam a visão de Oliveira e Godoy (2015), segundo as quais os estudos sobre resiliência diferem quanto à população e aos contextos, 
características que torna essencial o estudo das características específicas de cada população, a fim de que os elementos-chave desse processo possam ser identificados e utilizados no mapeamento das intervençôes.

Posteriormente, a temática abordada em cada estudo foi categorizada, de modo que foi possível identificar cinco categorias principais. A primeira referiuse à construção de instrumentos, composta por 24 artigos, correspondente a $35,29 \%$ dos trabalhos analisados. Foram inclusos nessa categoria todos os estudos que envolveram a elaboração de instrumentos, testes, baterias, questionários e inventários para a avaliação da resiliência. $\mathrm{Na}$ segunda, que se relacionou à temática de risco e prevenção, foram identificados 19 trabalhos, que perfazem $27,94 \%$ do total. Nessa categoria, foram elencados os estudos nos quais, por meio da avaliação da resiliência, buscou-se compreender as condições possíveis de risco, e os potenciais de enfrentamento dos indivíduos.

A terceira categoria diz respeito à promoção da saúde mental $(n=17)$ e referese a $25 \%$ dos trabalhos analisados. Foram agrupados nessa categoria os estudos que envolveram a avaliação do potencial resiliente, como uma estratégia de enfrentamento a condições psiquiátricas. A quarta categoria referiu-se à resiliência comunitária e foi composta por sete artigos, os quais representam 10,29\% do total. Nesta foram inclusos os estudos voltados à avaliação da resiliência em comunidades que apresentam condições adversas ao desenvolvimento individual, assim como de sustento dessas comunidades. E, por fim, a quinta categoria identificada, composta por 1 trabalho, versou sobre a temática específica da avaliação da resiliência em militares.

Os resultados também foram analisados quanto ao tipo de estudo realizado pelos pesquisadores (teórico ou empírico). Dos 68 artigos selecionados, 60,29\% apresentaram delineamento metodológico empírico ( $n=41)$ e 39,71\% mostraramse de natureza teórica. A predominância de trabalhos empíricos corrobora os dados apresentados por Lopes e Martins (2011), ao afirmarem que, ainda que seja possível observar um grande número de trabalhos qualitativos voltados ao tema da resiliência, no que se refere à avaliação desse tema, os trabalhos empíricos têm apresentado maior número e têm produzido contribuições valiosas para a melhor compreensão acerca da mensuração desse atributo.

Em seguida, a fim de compreender como os pesquisadores têm aferido o potencial resiliente em seus trabalhos, realizou-se o levantamento dos instrumentos e técnicas utilizados. Um total de 55 diferentes métodos para avaliação da resiliência, listados na tabela 1 , foram identificados nas publicações, sendo possível observar que o uso de estratégias qualitativas para a avaliação da resiliência figura entre as estratégias mais utilizadas (Lopes \& Martins, 2011), 
representadas por entrevistas, histórias de vida e outros métodos, citados por Reppold et al. (2012) como medidas tradicionalmente utilizadas na temática.

Tais dados concordam com a revisão realizada por Muñoz (2007), o qual, após revisão de 30 artigos voltados à construção e, ou, busca por evidencias de validade de medidas de resiliência, apontou que as medidas se agrupavam em três grandes áreas: medidas objetivas (mais qualitativas, envolvendo a tarefa de completar histórias, oferecendo desfechos para as situações adversas e, ou, problemáticas), medidas psicométricas (tais como escalas) e medidas neurológicas (investigando a influência da genética na resiliência ou ainda examinando o resultado de eletroencefalogramas).

Nota-se que os pesquisadores internacionais fazem uso de diferentes técnicas para realizarem a mensuração do construto, avaliando-o de forma objetiva por meio de instrumentos explicitamente relacionados ao termo resiliência. Das 55 possibilidades de mensuração do construto, 10 instrumentos utilizam objetivamente o termo em seus títulos.

Tabela 1 - Instrumentos citados nos trabalhos internacionais analisados

\begin{tabular}{|c|c|c|}
\hline Instrumento & Frequência & $\%$ \\
\hline Wagnild \& Young Resilience Scale & 15 & 27,27 \\
\hline $\begin{array}{l}\text { Avaliação qualitativa (entrevistas, história de } \\
\text { vida e demais estratégias) }\end{array}$ & 12 & 21,82 \\
\hline Connor-Davidson Resilience Scale (CD-RISC) & 11 & 20,00 \\
\hline Resilience Scale for Children and Adolescents & 4 & 7,27 \\
\hline Beck Inventory & 2 & 3,64 \\
\hline Depression Anxiety Stress Scales & 2 & 3,64 \\
\hline Multidimensional Fatigue Inventory Scale & 1 & 1,82 \\
\hline Adolescent Resilience Questionaire & 1 & 1,82 \\
\hline $\begin{array}{l}\text { Alcohol Use Disorders Identification Test } \\
\text { (AUDIT) }\end{array}$ & 1 & 1,82 \\
\hline Baruth Protective Factors Inventory (BPFI) & 1 & 1,82 \\
\hline Berlin Social Support Scale & 1 & 1,82 \\
\hline Birleson Depression Self-rating scale (DSRS) & 1 & 1,82 \\
\hline $\begin{array}{l}\text { California Health Kids Survey Resilience } \\
\text { Assessment Module (HKRA) }\end{array}$ & 1 & 1,82 \\
\hline $\begin{array}{l}\text { Centre for Epidemiologic Studies Depression } \\
\text { Scale }\end{array}$ & 1 & 1,82 \\
\hline Childhood Abuse Experience Scale & 1 & 1,82 \\
\hline $\begin{array}{l}\text { Communities Advancing Resilience Toolkit } \\
\text { (CART) }\end{array}$ & 1 & 1,82 \\
\hline $\begin{array}{l}\text { Conjoint Community Resilience Assessment } \\
\text { Measurement (CCRAM) }\end{array}$ & 1 & 1,82 \\
\hline
\end{tabular}


Craig Handicap Assessment and Reporting

Technique

Davidson Trauma Scale (DTS)

Depression Scale 1

1,82

Dysexecutive Questionaire for Children

1,82

Early Life Stress Inventory

1,82

European Organization for Research and Treatment of Cancer Core Questionnaire (Version 3.0)

General Health Questionnaire (GHQ-12)

Generalized Self-Efficacy Scale

Health Scale

Maslach Burnout Inventory (MBI-GS)

1,82

Measure of marital satisfaction

1,82

Metacognition Self-Assessment Scale

1,82

Mother-Infant Touch Survey

Obsessive-Compulsive Inventory-Revised

Parenting Competence Scale

Parenting Stress Index [PSI]

Physical Contact Assessment

Positive and Negative Syndrome Scale (PANSS)

Purpose in Life Test

Resilience Enhancement Kit Design Application

Rosenberg Self-esteem Scale

Self-Assessment of Resilience and Anxiety (SARA)

Self-compassion Scale

Social Support Appraisals (SS-A)

Social-Emotional Assets

State-Trait Anxiety Inventory

Strengths and difficulties questionnaire parents version

Subjective Resilience Questionnaire' (SRQ)

Successful AGing Evaluation (SAGE)

The Level of Service Inventory/Case

Management Inventory (LS/CMI)

The Wechsler Abbreviated Scale of Intelligence (WASI)

WHO-5 Wellbeing Index

Youth Self Report

Fonte: elaborado pelas autoras. 
Assim como ressaltado na literatura científica, os dados mostraram que a mensuração da resiliência se centra, nos estudos analisados, em uma série de outras variáveis psicológicas, envolvidas diretamente ou não no construto (Reppold et al., 2012; Prince-Embury et al., 2017; Waaktaar \& Torgersen, 2010), por meio da utilização de instrumentos que não avaliam propriamente o construto em si, mas sim em 24 construtos adjacentes à resiliência, tais como depressão, ansiedade, trauma, estresse, saúde geral, fadiga, uso de álcool, suporte social, abuso na infância, autoeficácia, personalidade, orientação de vida, burnout, satisfação marital, metacognição, transtorno obsessivo-compulsivo, competência parental, contato físico, propósito de vida, autoestima, autocompaixão, aspectos socioemocionais, forças e dificuldades, inteligência.

Essa constatação confirma a opiniāo de Harland, Harrison, Jones e ReiterPalmon (2005), de que o problema da medida envolve o fato de que nem sempre ela se relaciona diretamente com o conceito de resiliência, podendo envolver outros aspectos considerados similares. Assim, tais dados apontam que, apesar da relevância da temática, há inúmeras lacunas a serem preenchidas, não apenas do ponto de vista conceitual (Brandão et al., 2011), mas também no que diz respeito à construção de instrumentos (Prince-Embury et al., 2017), assim como da eficácia e pertinência de tais instrumentos (Reppold et al., 2012; Waaktaar \& Torgersen, 2010), dada a necessidade da utilização de instrumentos de medida que apresentem evidências de validade, precisão, rápida aplicação e interpretação, tal como recomendado por Lopes e Martins (2011).

Após a identificação dos instrumentos utilizados nos artigos consultados, para mensuração da resiliência, em um total de dez, optou-se por buscar maiores informaçôes sobre cada um. Tomando-se o número de instrumentos encontrados, a situação aponta para a existência de poucos testes validados para avaliação da resiliência disponíveis na literatura, ainda que o interesse pelo construto venha se mostrando crescente (conforme verificado na análise do número de publicações) e reflita os esforços da área na prevenção de problemas e promoção da saúde mental, dentro do modelo de Psicologia Positiva (Gurgel, Plentz, Joly, \& Reppold, 2013).

Os achados vão ao encontro dos dados relatados em revisão internacional, de publicações que relatavam construção e, ou, validação de instrumentos para se mensurar resiliência, realizada por Ahern, Kiehl, Sole e Byers (2006). Foram identificados sete instrumentos: Baruth Protective Factors Inventory (BPFI), Brief-Resilient Coping Scale (BRCS), Adolescent Resilience Scale (ARS), Connor-Davidson Resilience Scale (CD-RISC), Resilience Scale for Adults (RSA), Resilience Scale (RS) e Resilience Factors Scale (FSR). Similarmente, Gurgel et al. (2013) identificaram 13, incluindo nos instrumentos anteriormente 
citados a The Brief Resilience Scale (BRS), 10-item CD-RISC, Spanish Version of the Resilience Scale, Strong Souls, Military Social Health Index (MSHI), Deployment Risk and Resilience Inventory (DRRI), Deployment Risk and Resilience Inventory in French-Canadian Veterans, Patient-based HealthRelated Quality of Life Questionnaire in Schizophrenia e a Chinese Positive Youth Development Scale (CPYDS), sendo importante salientar que oito deles avaliavam diretamente a resiliência e, em outros cinco, tal construto era tomado como um dos fatores que compunham os construtos investigados. Baasch, Amorim e Cruz (2015) acrescentam ainda a Sabbag Resilience Scale (ERS), a Ego-Resilience Scale, Dispositional Resilience Scale (DRS) e a Escala dos Pilares de Resiliência (EPR).

Dos instrumentos citados, quatro encontram-se adaptados e validados para a população brasileira, a Resilience Scale, desenvolvida por Wagnild e Young (1993), com base em estudos voltados à investigação da sua estrutura interna (Pesce et al., 2005) e sua aplicabilidade no contexto organizacional (Oliveira \& Batista, 2008), a Connor-Davidson Resilience Scale desenvolvida por Connor e Davidson (2003) e investigada por Lopes e Martins (2011) em relação à adaptação teórica e semântica dos itens, estrutura interna e precisão, e a Resilience Scale for Adults (RSA), desenvolvida por Hjemdal, Friborg, Martinussen e Rosenvinge (2001), foi adaptada para o português brasileiro por Hjemdal, Roazzi, Dias, Roazzi e Vikan (2009). Estudos voltados à investigação de sua estrutura interna e consistência interna foram conduzidos por Carvalho, Teodoro e Borges (2014).

Já a EPR (Cardoso \& Martins, 2013) encontra-se disponível para uso comercial, estando aprovada pelo Conselho Federal de Psicologia, tendo apresentado evidências de validade, precisão e normatização para a população brasileira. Tal dado confirma a opinião de Gurgel et al. (2013), de que existem poucas investigações voltadas à adaptação brasileira dos testes internacionais envolvendo a avaliação da resiliência, de modo que o desenvolvimento de instrumentos nacionais ou adaptação dos internacionais devem ser incentivados, dada a carência de instrumentais observada.

Convém destacar, no entanto, que importante levantamento dos testes possíveis de serem usados como ferramentas auxiliares na identificação de aspectos relacionados à resiliência, ainda que não específicos para esse construto, apontou uma ampla gama de instrumentos aprovados para uso profissional e comercial (Knorst, 2012). Entre eles, fatores de risco (sob a forma de testes de personalidade e psicopatologias), fatores de proteção (por meio de instrumentos de personalidade, de relaçôes familiares, de fatores ambientais e sociais) podem ser avaliados fazendo-se uso de instrumentos disponíveis. 
Dado o foco de interesse da pesquisa, na investigação da temática dentro da área da avaliação psicológica, uma análise mais detalhada desses foi realizada. Para isso, buscaram-se, nos artigos analisados, as referências originais desses instrumentos específicos. Foi possível acessar, por meio de bases de dados eletrônicas, os artigos que apresentavam o processo de construção e de evidências de validade de tais instrumentos. Tais dados podem ser observados no quadro 1 e incluíram dados referentes ao nome do instrumento, autoria, população-alvo, tipo de instrumento, objeto de avaliação e tipo de estudo psicométrico.

\section{Quadro 1 - Instrumentos para avaliação da resiliência}

\begin{tabular}{c|c|c|c|c|c}
\hline $\begin{array}{c}\text { Nome do } \\
\text { instrumento }\end{array}$ & Autoria & $\begin{array}{c}\text { População- } \\
\text { alvo }\end{array}$ & Tipo & $\begin{array}{c}\text { Objeto de } \\
\text { avaliação }\end{array}$ & $\begin{array}{c}\text { Tipo de estudo } \\
\text { psicométrico }\end{array}$ \\
\hline
\end{tabular}

The Resilience Wagnil e Scale Young (1993) Adultos

Constantine, Benard e Crianças Diaz (1999)

California Healthy Kids Resilience Assessment

SelfBarnett,

Assessment of Connor e Resilience and Davidson Adultos (2001)

Avaliar níveis de adaptação psicossocial Grupo critério e positiva em análise fatorial condições, confirmatória situações e eventos adversos.

Escala de autorrelato do tipo Likert de 4 pontos.

Escala de autorrelato
Avaliar aspectos protetivos e de risco, num modelo multifatorial de resiliência.

Avaliar serenidade, confiança, bemestar físico, habilidades sociais, clareza mental e resiliência.
Análise do coeficiente $\alpha$ de Cronbach e análise fatorial confirmatória

Grupo critério, estudos de validade convergente, teste e reteste, análise de coeficiente $\alpha$ de Cronbach, sensibilidade à intervenção e análise fatorial confirmatória 
Escala de

Resiliência

de Connor-

Davidson

(CD-RISC) /

The Connor-

Davidson

Resilience

Scale

The Resilience

Scales for

Children and

Adolescents

Resilience

Enhancement

Kit Design

Application

Kruger e

Prinsloo

(2008)

Gartland,

Adolescent

Resilience

Questionnaire

Bond,

Olsson,

Buzwell

e Sawyer

(2011)

Conjoint

Community

Resilience

Assessment

Measurement

(CCRAM)

Embury
2006; 2010)

\section{Crianças e} adolescentes

Escala de autorrelato do tipo Likert de 5 pontos.

Medida

qualitativa

associada à

Resilience Scale

Escala de

autorrelato do

tipo Likert de 5

pontos.

Escala de

autorrelato do

tipo Likert de 5

pontos.
Mensurar

o potencial

resiliente de um

indivíduo exposto

a uma experiência

traumática

que possa

desencadear

respostas típicas

de quadros

depressivos,

ansiosos e de

estresse pós-

traumático.

\section{Avaliar}

características

positivas

pessoais e

vulnerabilidades,

baseadas

num modelo

multifatorial de

resiliência.

Confiabilidade por

meio da análise

do coeficiente

$\alpha$ de Cronbach,

análise fatorial

exploratória e

análise fatorial

confirmatória

Identificar,

desenvolver e

fazer o manejo

de habilidades

relacionas à

resiliência.

Avaliar

características

individuais e

sociais que

potencialmente

oferecem

uma resposta

resiliente.

Avaliação em

grupo controle

e experimental,

análise do

coeficiente $\alpha$

de Cronbach, teste e reteste e

análise fatorial

confirmatória

Pré e pós-teste

Análise do

coeficiente $\alpha$

de Cronbach e

análise fatorial

confirmatória

Busca avaliar

a habilidade

de diferentes

comunidades

em lidarem com

situações críticas.
Análise fatorial confirmatória, análise fatorial confirmatória, correlação de Pearson e análise multivariada da variância 
Subjective

Resilience

Questionnaire

(SRQ)
Alonso-

Tapia, Nieto Adolescentes

e Ruíz (2013)

$\begin{array}{ll} & \text { Questionário } \\ \text { Adolescentes } & \begin{array}{l}\text { com respostas } \\ \text { em modelo } \\ \text { Likert de } 5 \\ \text { pontos }\end{array}\end{array}$

Questionário com perguntas demográficas, experiências em situações de calamidade e resiliência comunitária
Avaliar respostas

positivas e reações negativas diante da adversidade experimentada por professores, pais e pares.

Busca avaliar a capacidade resiliente de comunidades diante de situações de calamidade e de adversidades.
Análise fatorial confirmatória, MANOVA, análise do coeficiente $\alpha$ de Cronbach e estudos de correlação e regressão

Teste de Wilcoxon, análise do coeficiente $\alpha$ de Cronbach e correlação de Pearson

\section{Fonte: elaborado pelas autoras.}

Observou-se que os instrumentos específicos de resiliência são, em sua maior parte, escalas do tipo autorrelato, a serem respondidas sob a forma de escalas Likert (entre 4 e 7 pontos). Duas delas foram publicadas na década de 1990, 4 na década de 2000 e 4 nos últimos cinco anos. Como foco, voltam-se à avaliação de aspectos, habilidades e características pessoais associadas à resiliência, fatores de risco/vulnerabilidade e proteção, bem como respostas resilientes, dentro de modelos multifatoriais do construto. Têm, como população-alvo, adultos $(n=3)$, adolescentes $(\mathrm{n}=3)$, crianças $(\mathrm{n}=1)$, adolescentes e crianças $(\mathrm{n}=1)$ e comunidades $(\mathrm{n}=2)$. Nenhum instrumento voltou-se à investigação da resiliência em idosos, sendo também empobrecidas as possibilidades de avaliação em crianças.

Convém destacar que tal dado contrasta com aqueles publicados por Fontes e Neri (2015), as quais, após revisão dos estudos nacionais e internacionais, encontraram 53 artigos que tinham como foco a resiliência e velhice. Ressaltase, no entanto, que a categoria com menor frequência de trabalhos publicados $(\mathrm{n}=4)$ foi aquela relacionada a medidas de resiliência nessa faixa etária, tendo sido possível verificar o uso da Connor-Davidson Resilience Scale (CD-Risk), Resilience Scale e The Brief Resilience Scale (BRS), ainda que, segundo as autoras, a escassez de pesquisas nacionais sobre resiliência em idosos ainda se faça presente. 


\section{CONSIDERAÇÕES FINAIS}

É possível reconhecer que, há mais de 40 anos, o tema da resiliência tem sido investigado por profissionais da Psicologia (Prince-Embury et al., 2017) e, embora o termo tenha sofrido diversas mudanças ao longo do tempo, notase que o tema tem-se mostrado relevante tanto para a pesquisa quanto para a prática (Yunes, 2003). Diante da relevância desse tema e de seu potencial para contribuir com o avanço do conhecimento de aspectos voltados ao enfrentamento das adversidades (Sapienza \& Pedromônico, 2005), enfermidades (Greco et al., 2006), ou ainda, no desenvolvimento de habilidades individuais e de suporte social (Junqueira \& Deslandes, 2003; Masten, 2001), uma revisão bibliográfica da produção científica voltada ao tema foi conduzida. Teve, como foco principal, a questão da avaliação desse construto, notadamente diante das recomendações de diferentes profissionais (Infante, 2007; Masten, 2001, Reppold et al., 2012; Waaktaar \& Torgersen, 2010), referentes aos cuidados necessários ao realizar-se a avaliação da resiliência.

Ao longo deste estudo, pôde-se observar que a resiliência se caracteriza como um tema controverso e com consideráveis lacunas referentes a aspectos teóricos, conceituais, e relacionado ao processo de mensuração dessa característica. Entretanto a resiliência também se mostra como relevante e necessária para os pesquisadores em Psicologia, assim como se nota também o crescimento do interesse de pesquisadores no que tange à questão da avaliação da resiliência.

Considerando-se que, neste estudo, ao optar-se por realizar a análise dos dados com base em palavras-chave bastante específicas, é possível que estudos que tratam da avaliação da resiliência de forma indireta não tenham sido contemplados, sendo essa uma das limitações da revisão realizada. Nesse mesmo sentido, é preciso retomar que o termo inicialmente utilizado pelos pesquisadores era o de "invulnerabilidade" (Brandão et al., 2011), termo que não foi usado nas buscas. Portanto é possível que as primeiras publicaçôes tenham sido realizadas empregando esse descritor, não tendo sido, portanto, localizadas nas buscas. Ressalta-se, ainda, como limitação dos dados analisados, o fato de que muitas bases de dados se tornaram eletrônicas somente nos últimos anos.

Ao optar pela busca por bases de dados digitais, muitos trabalhos podem ter sido excluídos dos resultados. Assim, o número das publicações dos últimos 15 anos, período compreendido na análise apresentada, provavelmente deve ser bem maior do que as que foram aqui analisadas. Outra limitação que deve ser considerada, ao longo da interpretação dos dados, refere-se ao uso dos instrumentos e seus respectivos públicos-alvo. É possível que instrumentos originalmente desenvolvidos para fins de avaliação de uma população específica 
também comecem a ter seu uso ampliado para outras faixas etárias, de modo que, por exemplo, instrumentos cujas publicações voltaram-se, no período investigado, à avaliação da resiliência na população adulta, também venham sendo, mais recentemente, investigados com a população de idosos. Considerando-se que a busca se encerrou no início do ano de 2016, um dos limites da análise envolve o fato de que trabalhos com essas características podem não ter sido contemplados, ainda que os instrumentos disponíveis para avaliação em adultos, sobretudo nacionais, mostrem-se escassos (Gurgel et al., 2013).

Diante dessas limitações, as quais restringem a possibilidade de generalização dos resultados, sugere-se a condução de outros estudos, a fim de que as compreensões aqui expostas possam ser ampliadas. Sugere-se ainda que outras bases de dados sejam utilizadas em pesquisa futuras, ampliando-se a busca para outras bases não contempladas, a fim de que os dados aqui relatados possam ser confirmados e expandidos. Espera-se que os resultados possam ser utilizados para a identificação de lacunas que necessitem de maiores investigaçôes, assim como apontar para as contribuições, necessidades e déficits da produção referente à resiliência no contexto internacional (Ferreira, 2002; Lustoza et al., 2010). Ressalta-se também a necessidade de que estudos com propósitos semelhantes sejam conduzidos junto a publicações nacionais, a fim de que um quadro mais amplo possa ser traçado, permitindo a comparação entre o desenvolvimento da área no Brasil e nos demais países bem como a identificação das lacunas que ainda se encontram presentes nos dois contextos. 


\section{REFERÊNCIAS}

Ahern, N. R., Kiehl, E. M., Sole, M. L., \& Byers, J. (2006). A review of instruments measuring resilience. Issues in Comprehensive Pediatric Nursing, 29, 103-125.

Alonso-Tapia, J., Nieto, C., \& Ruíz, M. A. (2013). Measuring subjective resilience despite adversity due to family, peers and teachers. Spanish Journal of Psycology, 16(e19), 1-13.

Baasch, D., Amorim, L., \& Cruz, R. M. (2015). Qualidades psicométricas de instrumentos de resiliência para adultos. Revista Borges: Estudos Contemporâneos em Ciências Sociais Aplicadas, 5(1), 38-53.

Barnett, S. D., Connor, K. M., \& Davidson, J. R. T. (2001). The self-assessment of resilience and anxiety: psychometric properties. CNS Spectrums, 6(1), 854857.

Brandão, J. M., Mahfoud, M., \& Gianordoli-Nascimento, I. F. (2011). A construção do conceito de resiliência em psicologia: discutindo as origens. Paidéia, 21(49), 263-271.

Cardoso, T., \& Martins, M. C. F. (2013). Escala dos pilares da resiliência. São Paulo: Vetor.

Carvalho, V. D., Teodoro, M. L. M., \& Borges, L. O. (2014). Escala de resiliência para adultos: aplicação entre servidores públicos. Avaliação Psicológica, 13(2), 287-295.

Castillo, J. A. G., Castillo-López, A. G., López-Sánchez, C., \& Dias, P. C. (2016). Conceptualización teórica de la resiliencia psicosocial y su relación con la salud. Health and Addictions, 16(1), 59-68.

Connor, K. M., \& Davidson, J. R. T. (2003). Development of a new resilience scale: the Connor-Davidson resilience scale (CD-RISC). Depression and Anxiety, 18(1), 71-82.

Constantine, N.A., \& Benard, B. (2001). California Healthy Kids Surveyassessment module: technical report. Berkeley: Public Health Institute. Recuperado a partrir de https://www.cde.ca.gov/ls/he/at/chks.asp

Ferreira, N. S. A. (2002). As pesquisas denominadas estado da arte. Educação e Sociedade, 23(79), 257-272. 
Fontes, A. P. (2010). Resiliência, segundo o paradigma do desenvolvimento ao longo da vida (life-span). Kairós, 7(1), 8-20.

Fontes, A. P., \& Neri, A. L. (2015). Resiliência e velhice: revisão de literatura. Ciência \& Saúde Coletiva, 20(5), 1475-1495.

Gartland, D., Bond, L., Olsson, C. A., Buzwell, S., \& Sawyer, S. M. (2011). Development of a multi-dimensional measure of resilience in adolescents: the adolescent resilience questionnaire. BMC Medical Research Methodology, 11(1), 1-10.

Gloria, C. T., \& Steinheardt, M. A. (2014). Relationships among positive emotions, coping, resilience and mental health. Stress and Health, 32, 145-156.

Godoy, K. A. B., Joly, M. C. R. A., Piovezan, N. M., Dias, A. S., \& Silva, D. V. (2010). Avaliação da resiliência em escolares do ensino médio. Mudanças Psicologia da Saúde, 18(1-2), 79-90.

Greco, C., Morelato, G., \& Ison, M. (2006). Emociones positivas: una herramienta psicológica para promocionar el proceso de resiliencia infantil. Psicodebate: Psicologia, Cultura y Sociedad, 7(1), 81-94.

Gurgel, L. G., Plentz, R. D. M., Joly, M. C. R. A., \& Reppold, C. T. (2013). Resiliência em adultos e idosos: revisão de instrumentos. Estudos de Psicologia, 30(4), 487-496.

Harland, L., Harrison, W., Jones, J. R., \& Reiter-Palmon, R. (2005). Leadership behaviors and subordinate resilience. Journal of Leadership \& Organizational Studies, 11, 2-14.

Hjemdal, O., Friborg, O., Martinussen, M., \& Rosenvinge, J. H. (2001). Preliminary results from the development and validation of a Norwegian scale for measuring adult resilience. Journal of the Norwegian Psychological Association, 38, 310-317.

Hjemdal, O., Roazzi, A., Dias, M. G. B. B., Roazzi, M., \& Vikan, A. (2009). Exploring the psychometric properties of the resilience scale for adults in a Brazilian sample. In D. Elizur, \& E. Yaniv (Orgs.), Facet new horizons in theory construction and data analysis. (pp. 120-138). Jerusalem: FTA.

Infante, F. (2007). A resiliência como processo: uma revisão da literatura recente. In A. Melillo, \& E. N. S. Ojeda (Orgs.), Resiliência: descobrindo as próprias fortalezas. (pp. 23-28). Porto Alegre: Artmed. 
Junqueira, M. F. P. S., \& Deslandes, S. F. (2003). Resiliência e maus-tratos à criança. Cadernos de Saúde Pública, 19(1), 227-235.

Knorst, C. E. K. (2012). Resiliência: instrumentos de avaliação no contexto brasileiro. (Monografia de Especialização). Universidade Federal do Rio Grande do Sul, Instituto de Psicologia, Porto Alegre. Recuperado a partir de https://www.lume.ufrgs.br/bitstream/handle/10183/40112/000826643. pdf?sequence $=1$

Krentzman, A. R. (2013). Review of the application of Positive Psychology to substance use, addiction, and recovery research. Psychology of Addictive Behaviors, 27(1), 151-165.

Kruger, L., \& Prinsloo, H. (2008). The appraisal and enhancement of resilience modalities in middle adolescents within the school context. South African Journal of Education, 28(1), 241-259.

Leykin, D., Lohad, M., Cohen, O., Goldberg, A., \& Aharonson-Daniel, L. (2013). Conjoint community resilience assessment measure-28/10 items (CCRAM28 and CCRAM10): a self-report tool for assessing community resilience. American Journal of Community Psychology, 52(1), 313-323.

Lopes, V. R., \& Martins, M. C. F. (2011). Validação factorial da escala de resiliência de Connor-Davidson (Cd-Risc-10) para brasileiros. Psicologia: Organizaçôes e Trabalho, 11(2), 36-50.

Lustoza, R. Z., Oliveira, K. L., \& Mello, B. N. (2010). Produção científica no contexto psicanalítico. Psico-USF, 15(2), 161-169.

Luthar, S., Cicchetti, D., \& Becker, B. (2000). The construct of resilience: a critical evaluation and guidelines for future work. Child Development, 71(3), 543-558.

Masten, A. S. (2001). Ordinary magic: resilience processes in development. American Psychologist, 56(3), 227-238.

Masten, A. S. (2014). Global perspectives on resilience in children and youth. Child Development, 85, 6-20.

Méis, L., \& Leta, J. (1996). O perfil da ciência brasileira. Rio de Janeiro: UFRJ.

Muñoz, D. E. O. (2007). La medición de la resiliencia. Investigación y Educación en Enfermería, 25, 58-65. 
Oliveira, A. F., \& Batista, R. L. (2008). Validação da escala de resiliência para o contexto organizacional. In Anais, 3 Congresso Brasileiro de Psicologia Organizacionale do Trabalho, Florianópolis. Florianópolis: Associação Brasileira de Psicologia Organizacional e do Trabalho, UFSC.

Oliveira, A. L., \& Godoy, M. M. C. (2015). O processo de resiliência do jovem aprendiz e as estratégias de conciliação estudo-trabalho. Boletim de Psicologia, 65(143), 175-191.

Pacico, J. C., \& Bastianello, M. R. (2014). As origens da Psicologia Positiva e os primeiros estudos brasileiros. In C. S. Hutz (Org.), Avaliação em Psicologia Positiva. (pp. 13-22). Porto Alegre: Artmed.

Pesce, R. P., Assis, S. G., Avanci, J. Q., Santos, N. C., Malaquias, J. V., \& Carvalhaes, R. (2005). Adaptação transcultural, confiabilidade e validade da escala de resiliência. Cadernos de Saúde Pública, 21, 436-448.

Pesce, R. P., Assis, S. G., Santos, N., \& Oliveira, R. V. C. (2004). Risco e proteção: em busca de um equilíbrio promotor de resiliência. Psicologia: Teoria e Pesquisa, 20(2), 135-143.

Pfefferbaum, R. L., Pfefferbaum, B., Nitiéma, P., Houston, J. B., \& Van Horn, R. L. (2015). Assessing community resilience: an application of the expanded CART survey instrument with affiliated volunteer responders. American Behavioral Scientist, 59(2), 181-199.

Poletto, M., Wagner, T. M. C., \& Koller, S. H. (2004). Resiliência e desenvolvimento infantil de crianças que cuidam de crianças: uma visão em perspectiva. Psicologia: Teoria e Pesquisa, 20(3), 241-250.

Prince-Embury, S. (2006). Resiliency scales for adolescents: a profile of personal strengths. San Antonio: Harcourt Assessment.

Prince-Embury, S. (2010). Psychometric properties of the scales for resiliency for children and adolescents and use for youth with psychiatric disorders. Journal of Psychoeducational Assessment, 28(4), 291-302.

Prince-Embury, S., Saklofske, D. H., \& Keefer, K. V. (2017). Tree-factor model of personal resilience. In U. Kumar (Ed.), The routledge international handbook of psychosocial resilience. (pp. 118-141). New York: Taylor \& Francis Group.

Reppold, C. T., Mayer, J. C., Almeida, L. S., \& Hutz, C. S. (2012). Avaliação da resiliência: controvérsia em torno do uso de escalas. Psicologia: Reflexão e Critica, 25(2), 248-255. 
Sapienza, G., \& Pedromônico, M. R. M. (2005). Risco, proteção e resiliência no desenvolvimento da criança e do adolescente. Psicologia em Estudo, 10(2), 209-216.

Seligman, M., \& Csikszentmihalyi, M. (2000). Positive Psychology: an introduction. American Psychologist, 55(1), 5-14.

Sims, K., \& Pooley, J. A. (2017). Posttraumatic growth amongst refugee populations. In U. Kumar (Ed.), The routledge international handbook of psychosocial resilience. (pp. 491-521). New York: Taylor \& Francis Group.

Souza, M. T. S., \& Cerveny, C. M. O. (2006). Resiliência psicológica: revisão da literatura e análise da produção científica. Revista Interamericana de Psicologia, $40(1), 115-122$.

Waaktaar, T., \& Torgersen, S. (2010). How resiliente are resilience scales?: the big five scales outperform resilience scales in predicting adjustment in adolescents. Scandinavian Journal of Psychology, 51(1), 157-163.

Wagnild G. M., \& Young H. M. (1993). Development and psychometric evaluation of the resilience scale. Journal of Nursing Measurement, 1(2), 165178.

Witter, G. P. (1999). Metaciência e leitura. In G. P. Witter (Org.), Leitura: textos e pesquisas. (pp. 13-22). Campinas: Alínea.

Yunes, M. A. M. (2003). Psicologia Positiva e resiliência: o foco no indivíduo e na família. Psicologia em Estudo, 8(1), 75-84.

Yunes, M. A. M. (2011). Psicologia Positiva e resiliência: foco no indivíduo e na família. In D. D. Dell'Aglio, S. H. Koller, \& M. A. M. Yunes (Orgs.), Resiliência e psicologia positiva: interfaces do risco a proteção. (pp. 45-68). São Paulo: Casa do Psicólogo. 